\title{
Discriminant Model of Coal Mining Microseismic and Blasting Signals Based on Waveform Characteristics
}

\author{
Baolin Li, ${ }^{1,2,3}$ Nan Li, ${ }^{1}$ Enyuan Wang, ${ }^{1,2,3}$ Xuelong Li, ${ }^{1,2,3}$ Zhibo Zhang, ${ }^{1,2,3}$ \\ Xin Zhang, ${ }^{1}$ and Yue Niu ${ }^{1,2,3}$ \\ ${ }^{1}$ State Key Laboratory of Coal Resources and Safe Mining, China University of Mining and Technology, Xuzhou, Jiangsu 221116, China \\ ${ }^{2}$ Key Laboratory of Gas and Fire Control for Coal Mines, China University of Mining and Technology, Xuzhou, Jiangsu 221116, China \\ ${ }^{3}$ School of Safety Engineering, China University of Mining and Technology, Xuzhou, Jiangsu 221116, China \\ Correspondence should be addressed to Nan Li; cumtlinan@126.com
}

Received 3 August 2017; Revised 12 October 2017; Accepted 24 October 2017; Published 4 December 2017

Academic Editor: Longjun Dong

Copyright (C) 2017 Baolin Li et al. This is an open access article distributed under the Creative Commons Attribution License, which permits unrestricted use, distribution, and reproduction in any medium, provided the original work is properly cited.

\begin{abstract}
Generally, there are two important types of microseismic (MS) signals caused by mining and blasting activities at coal mines. The waveform characteristics of MS signals using FFT, STA/LTA method, and envelope analysis were studied to distinguish these two types of MS signals. The main results are as follows: the dominant frequency and duration of two types of signals are significantly different. The following peak envelope curves of two types of MS signals fit a power function. The power exponent was obtained to describe the attenuated speed of the MS signals. The attenuation of the coal mining MS signals is slower and more fluctuant than that of the blasting signal. Waveform characteristics consisting of the dominant frequency, duration, and attenuation coefficient were extracted as the discriminating parameters. The discriminating performance of these parameters was compared and discussed. Based on the waveform characteristics, a discriminant model for coal mining MS and blasting signals was established by using Fisher linear discriminant method and its performance was checked. The results show that the accuracy of the discriminant model is more than $85 \%$, which can meet the requirements of MS monitoring at coal mines.
\end{abstract}

\section{Introduction}

The rockburst is a sudden, violent fracture of rock mass in tunnels and mines, generally caused by failure of highly stressed rock and the rapid instantaneous release of accumulated stain energy [1-3]. The rockburst often causes coal mass failure, roadways collapse, casualties, mining equipment, ground building damage, and so on. The occurrence of the rockburst is accompanied by many small energy microseismic activities which are of great importance for rockburst research [4]. A MS monitoring system installed in a mine can monitor the state of the rock mass by analyzing the MS events caused by the mining activities. The core parts of the MS monitoring system include the layout of the monitoring network, data processing, MS source location, and MS early warning [5]. One of the challenges of the data processing is to identify the MS signals caused by coal mining activities among the many interference signals [6-8]. In these complex waveforms, the blasting signals are dominant and can be easily confused with the coal mining MS signals. Thus, distinguishing between the coal mining MS signals and blasting signals becomes the main task of data processing and worth further study.

Recently, MS monitoring systems were widely installed in coal mines in China, and considerable MS data has been recorded. However, little attention has been paid to the discrimination of coal mining MS signals and blasting signals and there is also lack of related literature based on waveform analysis. By contrast, the discrimination of earthquake signals against those of nuclear explosions has been studied extensively. The widely used methods of discrimination can be divided into two categories. One is based on source or waveform characteristics which includes the source location, source depth, origin time, direction of first motion of $\mathrm{P}$ wave, ratio of surface wave magnitude to body wave magnitude, ratio of $\mathrm{P}$ wave first arrival amplitude to maximum amplitude, ratio of Love wave amplitude to Rayleigh wave amplitude, and ratio of $\mathrm{P}$ wave spectral amplitude to $\mathrm{S}$ wave spectral 
amplitude [9-11]. The other is based on statistical identification which includes Fisher discriminant classifier, logistic regression, unascertained measurement, and neural networks [12-14].

Studies on MS signals were also conducted in non-coal mines. Dong analyzed the source parameters of blasting and MS events of three mines in Australia and Canada and established three statistical discriminant models by the Fisher classifier, the naive Bayesian classifier, and the logistic Regression. The classification performances and discriminant precision of three statistical techniques were discussed and compared $[8,15]$. Ma et al. analyzed seven parameters to distinguish between mining MS signals and blasting signals in a phosphorite mine, based on which, two statistical discriminant models were proposed by the Bayesian classifier and the Fisher classifier. Their models were more accurate than traditional methods [16, 17]. Based on the works of Booker and Taylor, Malovichko applied a multivariate maximum-likelihood Gaussian classifier technique to discriminate between mining MS and blasting events. Analysis of a large number of events showed that approximately $20 \%$ (1431 out of 7053) of MS mining events were reclassified as blasting events [7]. Vallejos and Mckinnon applied logical regression and neural network modeling to classify mine earthquake and blasting events. The location error and 13 seismic parameters provided by full-waveform system of ESG were used to calibrate the models. The logical regression and neural network approaches can effectively classify different event categories under nonlinear conditions [18]. Zhao et al. decompose the signals in time and frequency domains, and time-frequency characteristics of two kinds of waveforms are analyzed. The results showed that the energy of MS signals was mainly concentrated in the lower frequency band than blast vibration signals [19]. Pan et al. proposed a comprehensive analysis method to identify and calibrate microseismic events by using STA/LTA method and waveform information [20]. Zhu et al. analyzed the fractal dimension box of specified frequency bands through the wavelet analysis and the fractal theory and obtained 23dimensional values of pattern recognition feature vector. Finally, a SVM network model was established to recognize MS events, but the efficiency of identification still needs further improvement [21]. Jiang et al. presented a strategy for classifying local multichannels MS waveforms, triggered by a single event. However, the method required a lot of computation and the efficiency of identification needs further improvement [22]. Li et al. analyzed the characteristics of coal mining MS and blasting signals using the Hilbert-Huang Transform (HHT) method, but, no clear line of demarcation between these characteristics of the two types of signals was obtained [23].

As the geological conditions in coal mine areas are complex and the transmission medium is not homogenous, the MS signals in coal mines are more complicated than those in non-coal mines and natural earthquakes. Thus, it is difficult to differentiate the two kinds of signals with high precision. Moreover, because most MS monitoring sensors used in coal mines are single component and the monitoring region is small, the difference between the propagation time of the $\mathrm{P}$ waves and $\mathrm{S}$ waves produced by the rupture of coal and rock is small. The $S$ waves are likely to overlap the $P$ coda waves, making it difficult to detect them. Therefore, the recognition method, utilizing the $\mathrm{P} / \mathrm{S}$-wave energy ratio which is widely used to identify nuclear explosion signals and natural earthquake signals, is not suitable for coal mines. In this paper we analyzed three significant characteristics of these two types of signals using the Fourier transform (FFT), short-term averaging/long-term averaging (STA/LTA), and envelope analysis. Based on the characteristics, a discriminant model for coal mining MS signals and blasting signals, with the help of Fisher linear discriminant method, is established, which are useful to improve the efficiency of automatic discrimination and reduce the workload of artificial discrimination.

\section{Outline of the Mine and MS Monitoring System}

2.1. Coal Mine and Field MS Site Description. The Qianqiu coal mine is located at Sanmenxia city, Henan province, China, with a history of rockburst. It is one of the core mines in the Yima Coal Group with the annual coal production of 2.1 million tons. The number 21 mining district was the main production area and the number 21141 working face was the main mining face when this work was conducted. As shown in Figure 1, the 21141 is a longwall panel in the number 21 mining district with a dimension of $1500 \mathrm{~m} \times$ $130 \mathrm{~m}$. The average overburden depth is $684.4 \mathrm{~m}$, and the average coal seam thickness and dip angle are $10.6 \mathrm{~m}$ and $13^{\circ}$, respectively. The number 21121 goaf and number 21161 virgin field are located in the northern and southern side of number 21141 longwall panel, respectively. The immediate roof of the coal seam is composed of dark grey mudstone with the thickness of $23.02-27.63 \mathrm{~m}$. The main roof is composed of mottled sand, conglomerates, and sandstone with an average thickness of $612 \mathrm{~m}$. The huge thick gravel roof can easily accumulate a great deal of elastic energy which can be released suddenly during the process of fracturing or sliding, leading to a rockburst. According to the field statistical analysis, 5 rockbursts occurred within $100-200 \mathrm{~m}$ of the working face and 9 rockbursts occurred more than $200 \mathrm{~m}$ from the working face from 2008 to 2012.

2.2. The MS Monitoring System. The rockbursts and mine earthquakes have become more and more severe with the increase of mining depth and intensity. To monitor these mining induced seismic activities, the ARAMIS M/E MS monitoring system was installed in the Qianqiu coal mine. This system was developed by EMAG mining automation center, Poland. MS signals are recorded by the SPI-70 microseismic sensors and transmitted these signals to the data analysis center by the digital transmission system (DTSS). The system can monitor events with energy greater than 100J, frequency less than $250 \mathrm{~Hz}$, and dynamic ranges less than $100 \mathrm{~dB}$. The MS technique has been a daily rockburst monitoring tool because of the real time monitoring capability, source location, events energy calculations, and rockbursts risk assessment. The ARAMIS M/E MS monitoring system at 


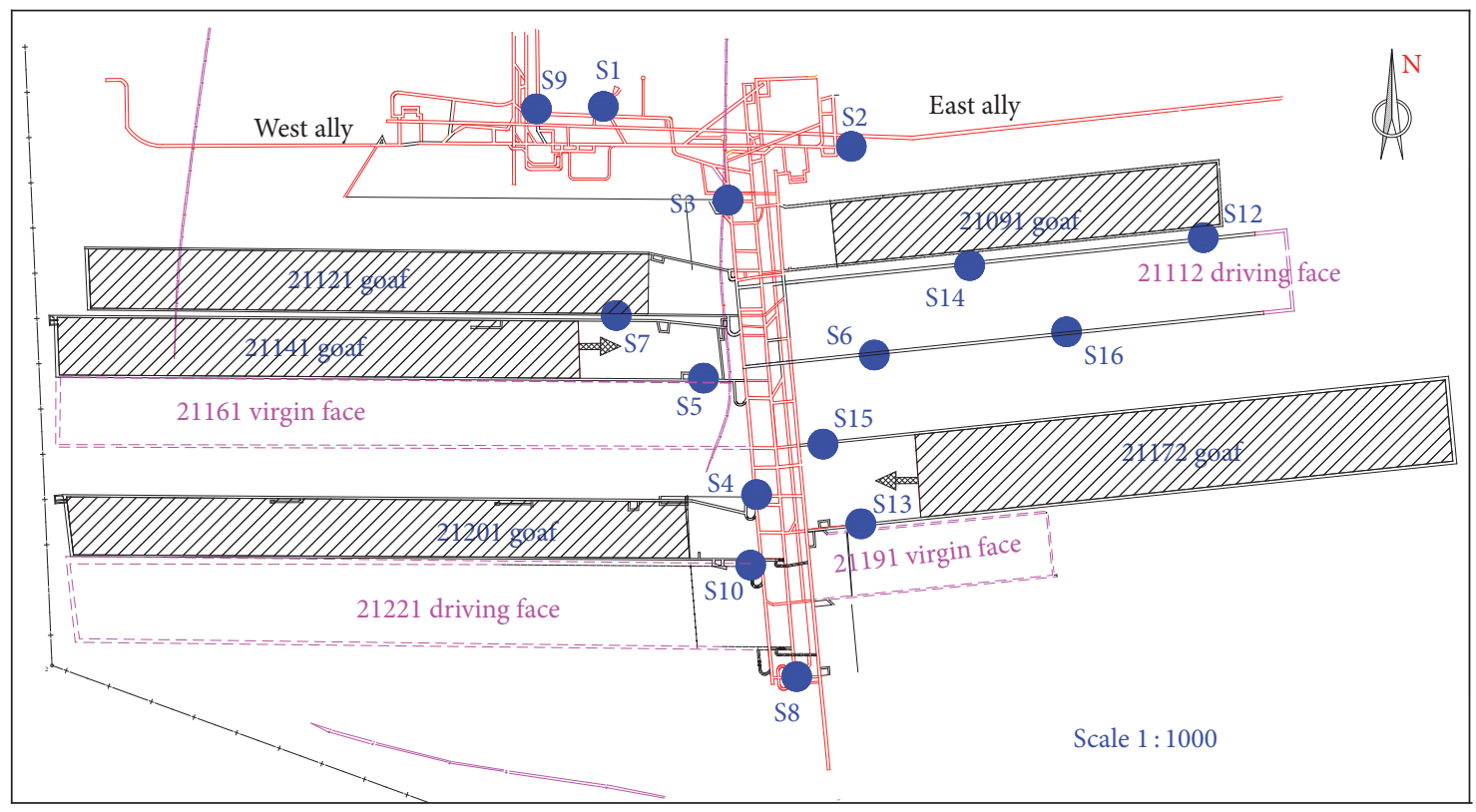

Microseismic sensor

FIGURE 1: Number 21141 longwall panel and layout of the ARAMIS M/E system sensors network.

the Qianqiu coal mine is equipped with 16 MS sensors, S1, S2, ..., S16. The layout of the MS monitoring network is shown in Figure 1.

2.3. Sample Database. The MS events recorded by the ARAMIS MS monitoring system can be divided into two main categories: (1) coal mining MS events, which refer to the vibration caused by mining; they contain abundant information on the fracturing of the coal and rock; (2) blasting events, which refer to the vibration caused by blasting. The type of recorded event was manually confirmed and marked with different symbols in the database of ARAMIS system. To establish discriminant model of coal mining MS and blasting signals, fifty coal mining MS events and fifty blasting events were randomly selected as the training sample; the other fifty coal mining MS events and fifty blasting events were selected as the testing sample. As the distance between sensors and hypocenter increases, the attenuation and changes of signals become stronger. Therefore, the earliest available signal, received by the nearest sensor from the source, should be selected as the representative signal of the event. Finally, a total of one hundred training sample signals and one hundred testing sample signals are obtained.

\section{Characteristics of the Coal Mining MS and Blasting Signals}

\subsection{Spectral Characteristics of the Coal Mining MS and Blasting Signals}

3.1.1. Fourier Transform Method. In the field of frequency spectrum analysis, the fast Fourier transform (FFT) is often used to convert signals from the time domain into the frequency domain $[24,25]$. The theoretical basis of FFT is as follows: any function that satisfies the Dirichlet condition can be expressed as a sum of sinusoidal functions of different frequencies. The proportion of sine waves of frequency $w$ in these signals is given by [26]

$$
F(w)=\int_{-\infty}^{\infty} f(t) e^{-j w t} d t
$$

3.1.2. Dominant Frequency Statistics for the Coal Mining $M S$ and Blasting Signals. For the signal processing in this work, the MATLAB command FFT was used to perform the frequency spectrum analysis. The dominant frequencies of 100 training sample signals (50 coal mining signals and 50 blasting signals) were obtained and analyzed statistically. As shown in Figure 2, the dominant frequencies lie in the band of below $150 \mathrm{~Hz}$. The majority of the coal mining MS signals fall within the low-frequency range while most of the blasting signals are in the high-frequency range. Below $80 \mathrm{~Hz}$ there are 39 signals, which are $78 \%$ of all the coal mining MS signals. Above $80 \mathrm{~Hz}$ there are 42 signals, comprising $84 \%$ of all the blasting signals. The results demonstrate that the dominant frequency of the coal mining MS signals is generally lower than that of the blasting signals. Thus the dominant frequency can be an important index for distinguishing the two types of signals.

\subsection{Duration of the Coal Mining MS and Blasting Signals}

3.2.1. Calculating the Signal Duration. The duration of the signals is the time from the arrival time to the end of undulations (Figure 3 ). We define $T_{\text {start }}$ as the arrival time and 


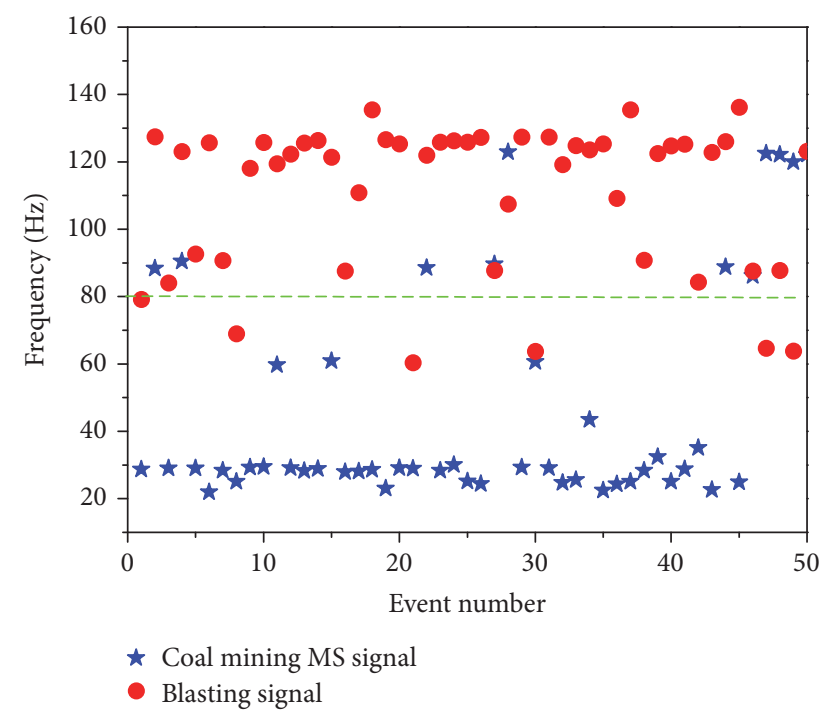

FIGURE 2: Distribution of the dominant frequency of coal mining MS and blasting signals.

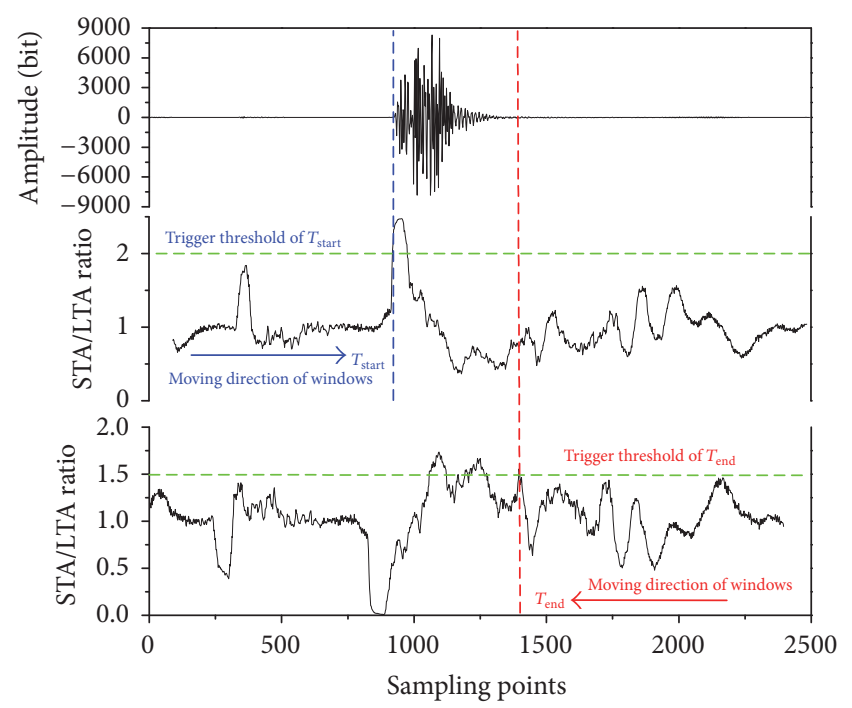

FIGURE 3: Diagram illustrating the arrival and termination times of a coal mining MS signal.

$T_{\text {end }}$ as the terminate time of a wave. For the sample frequency $f_{s}$, the duration is given by

$$
T_{L}=\frac{\left(T_{\text {end }}-T_{\text {start }}\right)}{f_{s}} .
$$

The arrival time and termination time can be determined by the short-term average/long-term average (STA/LTA) method as [27-29]

$$
R_{i}=\frac{\operatorname{STA}}{\operatorname{LTA}}(i)=\frac{n_{l}}{n_{s}} \frac{\sum_{j=i-n_{s}}^{i} \mathrm{CF}(j)}{\sum_{j=i-n_{l}}^{i} \mathrm{CF}(j)},
$$

where $i$ is the sampling moment, which represents the common position of short time window and long time window. $j$ ranges in each of short time and long time window, and

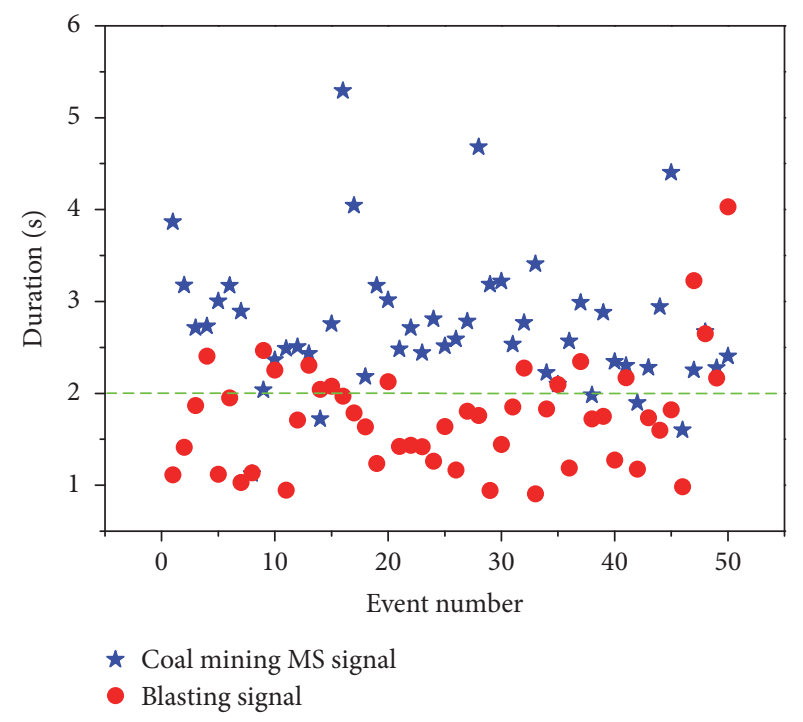

FIGURE 4: Duration distribution of coal mining MS and blasting signals recorded in the coal mine.

it is used to calculate average values of signal amplitude in the windows. $n_{l}$ is the length of the long time window, and $n_{s}$ is the length of the short time window. $\mathrm{CF}(i)$ represents the characterization function at point $i$. In practice, $\mathrm{CF}(i)$ is often replaced with the signal energy or the absolute value of amplitude. When the ratio $R_{i}>\lambda$ ( $\lambda$ is the trigger threshold), an event is detected. Thus, automatic recognition and arrival picking can be implemented. In this study, the values for $T_{\text {start }}$ are $n_{l}=50$ (sampling points), $n_{s}=20$ (sampling points), and $\lambda=2$ and the values for $T_{\text {end }}$ are $n_{l}=50$ (sampling points), $n_{s}$ $=20$ (sampling points), and $\lambda=1.5$.

\subsubsection{Duration Statistics of the Coal Mining MS and Blasting} Signals. Taking one of the coal mining MS signals as an example, the STA/LTA time series of the arrival time and terminate time are shown in Figure 3. The windows move from the first point to the last point for picking $T_{\text {start }}$, and the windows move from the last point to the first point for picking $T_{\text {end }}$. The coal mining MS signal starts at the 920th data point and ends at the 1398th data point in Figure 3. So the duration is $T=(1398-920) / 500=0.956 \mathrm{~s}$ according to (2).

Figure 4 shows the durations of 100 training sample signals. The duration distribution of all the signals is in the range of $0.5 \sim 6 \mathrm{~s}$; most of the coal mining MS signals last longer than the blasting signals. With $2 \mathrm{~s}$ as the dividing line, 44 signals lasted $2 \sim 6 \mathrm{~s}$, making up $88 \%$ of the coal mining signals and 35 signals lasted $0.5 \sim 2 \mathrm{~s}$, contributing $70 \%$ of the blasting signals. Therefore, the duration of the signal can be another important index to differentiate between the coal mining MS and blasting signals in coal mines.

\subsection{Attenuation Characteristics of the Coal Mining MS and Blasting Signals}

3.3.1. Calculating the Attenuation Coefficient. We defined the period from the peak point of the MS waveform (maximum 


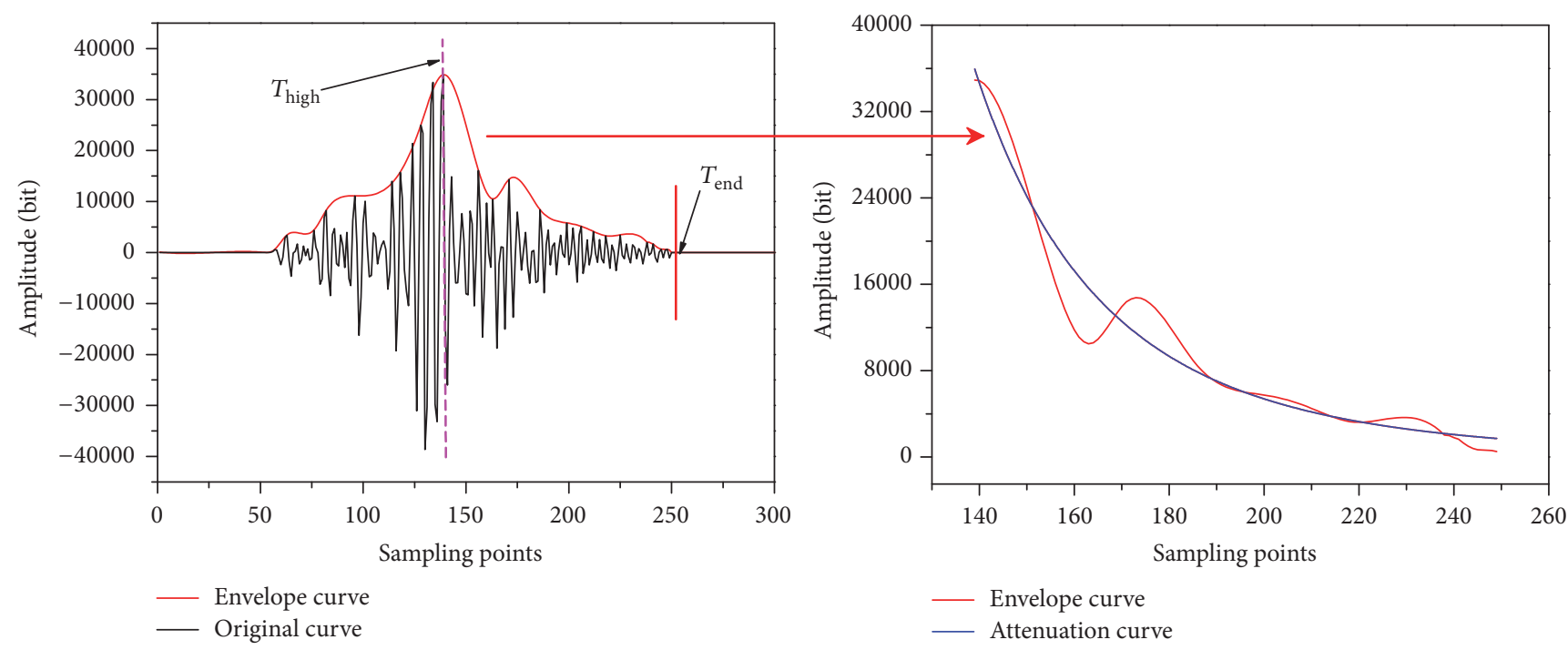

FIGURE 5: Following peak attenuation curve of typical coal mining MS signal.

amplitude) to the corresponding point of terminate time as the attenuation of the MS signal. The following peak attenuation curve is proposed for quantitative analysis of the MS signal attenuation. In Figure $5, T_{\text {high }}$ is the time of peak point, $T_{\text {end }}$ is the termination time, the envelope fitting curve from $T_{\text {high }}$ to $T_{\text {end }}$ is defined as the following peak attenuation curve.

To investigate the following attenuation curve, the envelope curve should be obtained first. The steps to obtain the envelope curve are as follows:

(1) Picking all the peaks of the waveform

(2) Interpolating the discrete peaks using cubic spline interpolation to obtain a complete envelope curve.

The cubic spline interpolation $[30,31]$ is based on the following method: the interval $[a b]$ is divided as $a \leq x_{0}<$ $x_{1}<\cdots<x_{n} \leq b$. For the point $x_{i}, y_{i}=f\left(x_{i}\right)$, if the function $S(x)$ is cubic polynomial in every small interval $\left[x_{i}, x_{i+1}\right]$ and also has second-order continuous derivatives in $[a, b], S(x)$ is regarded as a cubic spline function in $\left[\begin{array}{ll}a & b\end{array}\right]$. Moreover, if $S(x)$ satisfies

$$
S\left(x_{i}\right)=y_{i} \quad(i=0,1, \ldots, n)
$$

after derivation, the expression of $S(x)$ is

$$
\begin{array}{r}
S(x)=\alpha_{i}(x) y_{i}+\alpha_{i+1}(x) y_{i+1}+\beta_{i}\left(x_{i}\right) m_{i}+\beta_{i+1} m_{i+1}, \\
x \in\left[x_{i}, x_{i+1}\right] .
\end{array}
$$

To study the function form corresponding to the following peak envelope curve of the coal mining MS and blasting signals recorded in the mine and then to describe the signal attenuation process quantitatively, it is necessary to fit the following peak envelope curve of the signals. Gao et al. found that the shock vibration energy in rock-soil medium diminished in a power relation with respect to the travel distance [32]. This power function is adopted here to investigate the attenuation of the recorded signals:

$$
y=a x^{b}
$$

where $y$ is the amplitude of the signal, $x$ is the sampled point, and $a$ and $b$ are the fitting parameters corresponding to the peak value and attenuation rate, respectively. Generally speaking, the energy of the signal decreases more rapidly when the absolute of $b(|b|)$ increases; thus, $|b|$ is called the attenuation coefficient. The fitting precision is represented by the adjusted $R$-squared value, which is a calibration correlation coefficient and is widely used in Origin and MATLAB. A coefficient value close to 1 indicates a highfitting precision and stable attenuation of the signal's energy.

3.3.2. Statistics of Attenuation Coefficient and Fitting Precision. Taking the coal mining MS signal in Figure 3 as an example, the envelope curve of the waveform is given by the cubic spline interpolation and the termination time point is determined by the STA/LTA method. The fitted envelope curve stretching from the maximum amplitude point to the termination time point is shown in Figure 6. The attenuation coefficient of the signal is 27.73 with a fitting precision (Adj. $R$-square) of 0.96 , indicating that the following peak envelope curve satisfies the power function.

The fitting precision and attenuation coefficient of 100 training sample signals are obtained as shown in Figure 7. Figure 7 (a) shows the distribution of the fitting precision of the following peak attenuation curve. Of the 100 sampled signals, 93\% show a fitting precision above 0.8 , indicating that the attenuation curve of both types of signals satisfy the power function. The fitting precision of the following peak attenuation curve of the coal mining MS signals is mainly within $0.8 \sim 1$, with $42 \%$ between 0.8 and 0.9 and $48 \%$ between 0.9 and 1 . The fitting precision of the following peak attenuation curve of the blasting signal is higher, with $84 \%$ of the signals falling in the range of $0.9 \sim 1$. Therefore, in most 


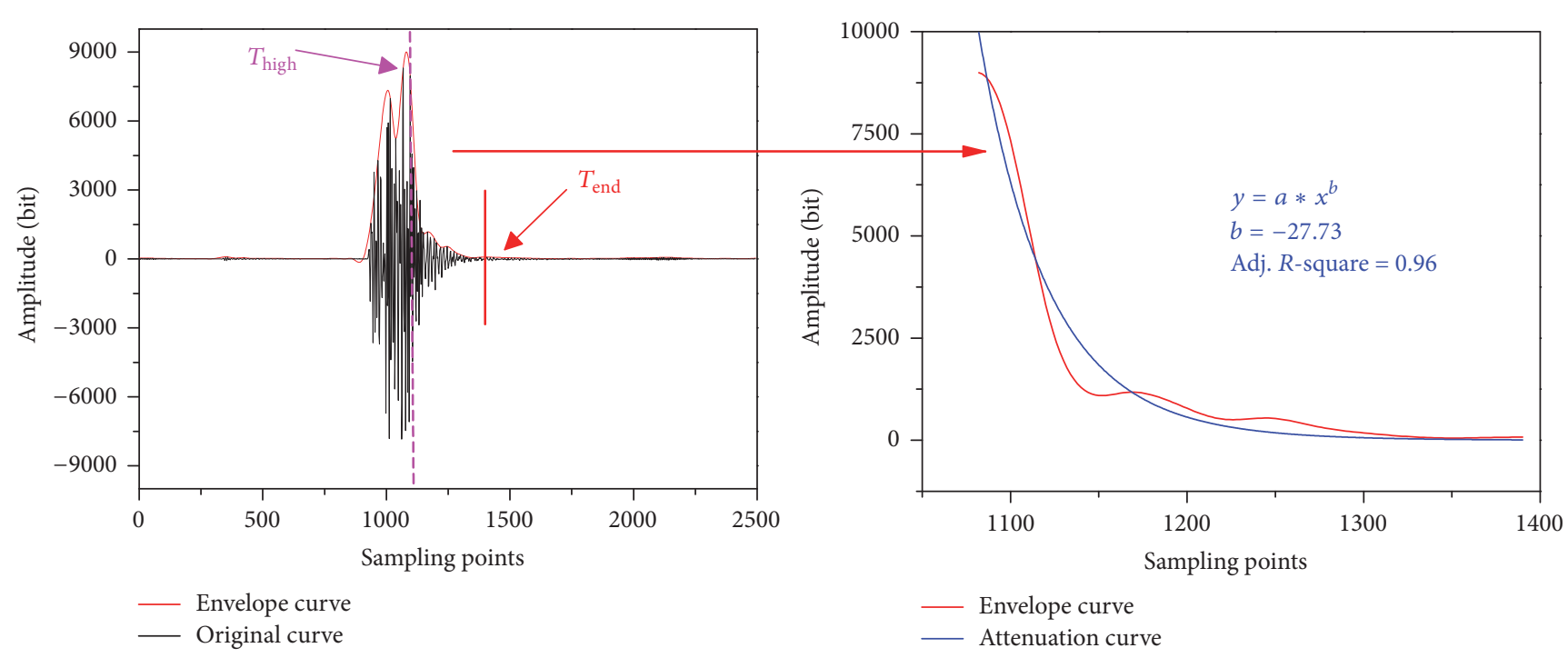

FIGURE 6: Envelope curve and following peak attenuation curve of a coal mining MS signal.

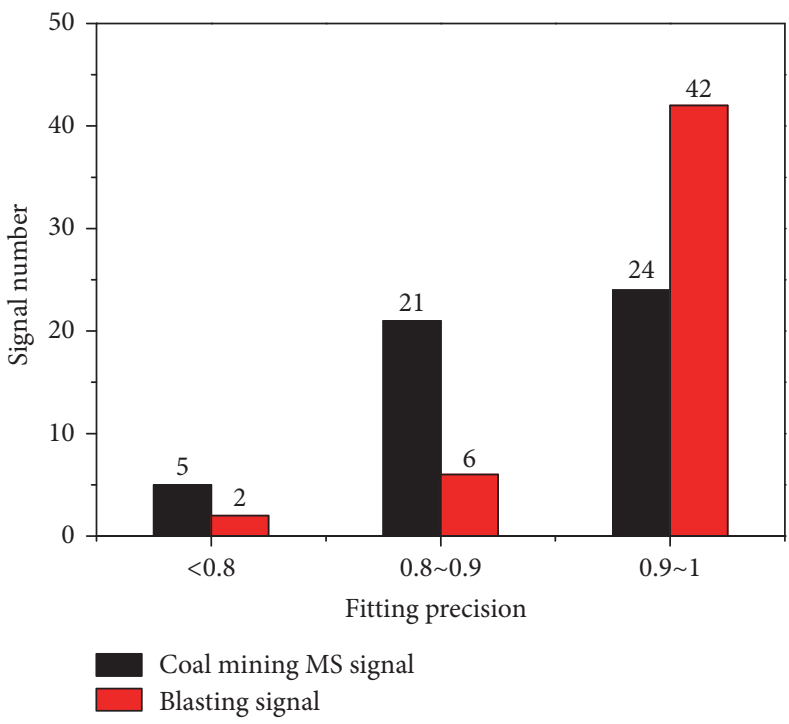

(a) Histogram of fitting precision

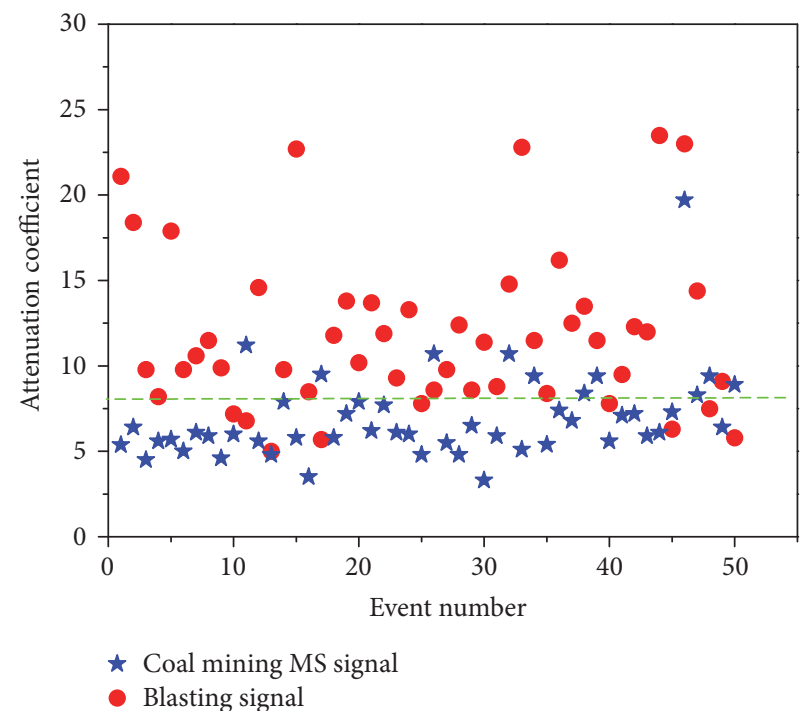

(b) Distribution of attenuation coefficient

FIGURE 7: Distribution of fitting precision and attenuation coefficient of all the signals.

cases, the fitting precision of the attenuation curve of the coal mining MS signals is lower than that of the blasting signals. The main reason is that the attenuation of the coal mining MS signals often fluctuates while the attenuation of the blasting signals is rapid and steady.

Figure 7(b) shows the distribution of the attenuation coefficient for the coal mining MS and blasting signals, which is generally within the range of $2 \sim 25$. The attenuation coefficients of the coal mining MS signals are smaller than those of the blasting signals, which demonstrates the slower damping of the coal mining MS signals. Among all the coal mining MS signals, the attenuation coefficients of 39 signals $(78 \%)$ are located in the range $2 \sim 8$. Among all the blasting signals, the attenuation coefficients of 35 signals (70\%) are located in the 8 25 range. This demonstrates that the attenuation coefficient can reflect the difference between the two kinds of signals and can be used as an important index to distinguish between the two types of signals.

\section{Discriminant Model of the Coal Mining MS and Blasting Signals}

4.1. Fisher Linear Discriminant. Pattern recognition is an emerging subject in the 1960s. After decades of research, it has been successfully used in various fields. For instance, it has been applied to analyze nuclear explosion and earthquakes and has achieved some results [12-14]. However, it is seldom applied in the discrimination of coal mining MS signals and blasting signals. Considering that there is a lack of the corresponding geophysical models and equations in the 
discrimination of coal mining MS signals and blasting signals and the data are not subject to the classical statistics, so the pattern recognition has become an effective research method. The pattern recognition methods include Bayes method, minimum distance method, ICHAM, Fisher, SVM, and BEG, in which Fisher linear discriminant analysis has been widely used in seismology. Therefore, this paper introduces the Fisher linear discriminant analysis for the discrimination of coal mining MS signals and blasting signals.

The basic principle of Fisher linear discriminant analysis is to project multidimensional and multiclass data to the same direction and separate two or more classes $[15,17]$. In fact, it is a dimension reduction process. Suppose the number of collectivity is $m$, that is, $G_{1}, G_{2}, \ldots, G_{m}$. The corresponding mean vectors and covariance matrices are $\mu^{(1)}, \mu^{(2)}, \ldots, \mu^{(m)}$ and $V^{(1)}, V^{(2)}, \ldots, V^{(m)}$, respectively. The number of $n_{i}$ samples is selected from the total samples, as follows [33, 34]:

$$
\begin{aligned}
X_{\alpha}^{(i)}=\left\{x_{\alpha 1}^{(i)}, x_{\alpha 2}^{(i)}, \ldots, x_{\alpha p}^{(i)}\right\}^{T} & \\
& \left(\alpha=1,2, \ldots, n_{i} ; i=1,2, \ldots, m\right) .
\end{aligned}
$$

$\mu^{T} X_{\alpha}^{(i)}$ is the projection of $X_{\alpha}^{(i)}$ on the axis, which is defined as

$$
\begin{aligned}
\mu^{T} X_{\alpha}^{(i)} & =\left\{\mu_{1} x_{\alpha 1}^{(i)}, \mu_{2} x_{\alpha 2}^{(i)}, \ldots, \mu_{p} x_{\alpha p}^{(i)}\right\}^{T} \\
\bar{X}^{(i)} & =\frac{1}{n_{i}} \sum_{i=1}^{n_{i}} X_{(\alpha)}^{(i)} \\
\bar{X} & =\frac{1}{n} \sum_{i=1}^{m} \sum_{\alpha=1}^{n_{i}} X_{(\alpha)}^{(i)} \\
n & =\sum_{i=1}^{m} n_{i},
\end{aligned}
$$

where $\bar{X}^{(i)}$ and $\bar{X}$ are the intergroup average and the total average, respectively. The intergroup difference is as follows:

$$
\begin{aligned}
e & =\sum_{i=1}^{m} \sum_{\alpha=1}^{n_{i}}\left(\mu^{T} X_{(\alpha)}^{(i)}-\mu^{T} \bar{X}^{(i)}\right)^{2} \\
& =\mu^{T}\left\{\sum_{i=1}^{m}\left[\sum_{\alpha=1}^{n_{i}}\left(X_{(\alpha)}^{(i)}-\bar{X}^{(i)}\right)\left(X_{(\alpha)}^{(i)}-\bar{X}^{(i)}\right)^{T}\right]\right\} \mu \\
& =\mu^{T}\left\{\sum_{i=1}^{m} S_{i}\right\} \mu \triangleq \mu^{T} W \mu \\
W & =\left\{\sum_{i=1}^{m} S_{i}\right\},
\end{aligned}
$$

where $\mu$ is the maximum eigenvalue $\lambda$ 's corresponding eigenvector and $S_{i}$ is the deviation matrix of $n_{i}$ samples
$X_{\alpha}^{(i)}\left(\alpha=1,2, \ldots, n_{i}\right)$ in $G_{i}$ samples. The intergroup difference is as follows:

$$
\begin{aligned}
b & =\sum_{i=1}^{m} \sum_{\alpha=1}^{n_{i}}\left(\mu^{T} \bar{x}^{(i)}-\mu^{T} \bar{x}\right)^{2} \\
& =\sum_{i=1}^{m} n_{i} \mu^{T}\left(\bar{X}^{(i)}-\bar{X}\right)\left(\bar{X}^{(i)}-\bar{X}\right)^{T} \mu \\
& =\mu^{T}\left[n_{i} \sum_{i=1}^{m}\left(\bar{X}^{(i)}-\bar{X}\right)\left(\bar{X}^{(i)}-\bar{X}\right)^{T} \mu\right] \triangleq \mu^{T} B \mu .
\end{aligned}
$$

To let the discrimination function better distinguish samples from different population samples, the difference between two groups from two population samples is expected to be bigger while the intergroup difference is expected to be smaller. Above all,

$$
\Phi=\frac{b}{e}=\frac{\mu^{T} B \mu}{\mu^{T} W \mu} .
$$

For this, the Lagrange's multiplier is applied and the equation of $F=\mu^{T} B \mu-\lambda\left(\mu^{T} W \mu-1\right)$ can be obtained. The partial differential result for $F$ is 0 :

$$
\frac{\partial F}{\partial \mu}=2 B \mu-2 \lambda W \mu \triangleq 0 .
$$

By calculating the above formula, the following result could be obtained.

$$
\left(W^{-1} B-\lambda I\right) \mu \triangleq 0 .
$$

Then the coefficients of the discrimination function could be calculated. $I$ is the ratio of sum of squares of deviation within the group to the sum of squares of deviation between groups. By solving the equations, $S-2$ discrimination functions could be obtained $(S=\min \{G-1, m\})$. Generally, most of the information of the sample could be explained by the first equation. If the first discrimination function is difficult to distinguish, the following discrimination functions could be applied for comprehensive consideration.

Writing the function $y=\mu^{T} X$ and plugging $\bar{X}^{(i)}$ into $y=$ $\mu^{T} X, \bar{y}^{(i)}$ could be obtained. Then values of $\bar{y}^{(i)}$ are ranged from small to large; for example, if $\bar{y}^{(1)}<\bar{y}^{(2)}<\cdots \bar{y}^{(m)}$, the threshold value between $G_{i}$ and $G_{i+1}$ is as follows:

$$
y_{c}(i, i+1)=\frac{n_{i} \bar{y}^{(i)}+n_{i+1} \bar{y}^{(i+1)}}{n_{i}+n_{i+1}} .
$$

Take the training sample data of the known type into the above formula and predict its type. For the given sample $X$, if $y=\mu^{T} X$ is between $y_{c}(i-1, i)$ and $y_{c}(i, i+1), X$ belongs to $G_{i}$.

4.2. Discriminant Model. To use Fisher linear discriminant analysis to get the discriminant function of coal mining MS and blasting signals and establish discriminant model, it is 
TABLE 1: Tests of equality of group means.

\begin{tabular}{lcccrr}
\hline Characteristic variables & Lambda of Wilks & $F$ & df1 & df2 & Sig. \\
\hline Dominant frequency & 0.427 & 131.674 & 1 & 98 & 98000 \\
Duration & 0.654 & 51.917 & 1 & 98 & 0.000 \\
Attenuation coefficient & 0.701 & 41.775 & 1 & 98 & 0.000 \\
\hline
\end{tabular}

TABLE 2: Structure matrix.

\begin{tabular}{lc}
\hline Characteristic variables & Correlation coefficient \\
\hline Dominant frequency & 0.796 \\
Duration & -0.500 \\
Attenuation coefficient & 0.448 \\
\hline
\end{tabular}

necessary to select proper signal characteristics to establish eigenvectors. According the discussion of third chapter, we can find that these two types of signals are significantly different in dominant frequency, duration, and attenuation coefficient. Therefore, these three characteristics are chosen to construct the eigenvector. The $100 \times 3$ characteristic matrix is composed of training sample signal's dominant frequency, duration, and attenuation coefficient. Meanwhile, the modeling needs one column of data to show the real type of signals in each group. Thus the coal mining MS signals are marked as 1 and the blasting signals are marked as 2 . Importing the characteristic matrix and categorical data of samples into SPSS to establish the model, partial results are as follows.

(1) Tests of Equality of Group Means. Table 1 shows the tests of equality of group means. It reflects these two types of signals' mean differences in different indexes. The probability $p$ (Sig.'s corresponding data in Table 1) is all less than 0.001 in the detection of the coal mining MS signals and blasting signals. It is indicated that there are significant differences between these two types of signals in each index, so the discriminant analysis could be carried out.

(2) Structure Matrix. The structure matrix in Table 2 shows signals' dominant frequency, duration, and attenuation coefficient and the final discriminant functions' correlations between groups. It could be seen that the bigger the correlation coefficient's absolute value, the greater the effects of the variable on these two types of signals' discriminant functions. As shown in the table, the dominant frequency has the greatest effects on the discriminant functions followed by duration. The attenuation coefficient's impact is minimal, but the correlation coefficient still reaches 0.448 .

(3) Central Values of the Final Discriminant Function in the Coal Mining MS and Blasting Signals. Table 3 shows the central values of the final discriminant functions in the coal mining MS signals and blasting signals. The result shows only one discriminant function, with a discriminant score of -1.442 and 1.442 in the center of coal mining MS signals and blasting signals, respectively. Therefore, when the signal's discriminant score is near -1.442 , the signal will belong to
TABLE 3: Central values of the final discriminant function in the coal mining MS signals and blasting signals.

\begin{tabular}{lc}
\hline Category of signal & Central values of the function \\
\hline Coal mining MS & -1.442 \\
Blasting & 1.442 \\
\hline
\end{tabular}

TABLE 4: Discriminant function coefficients of two types of signals.

\begin{tabular}{lc}
\hline Characteristic variables & Discriminant function coefficients \\
\hline Dominant frequency & 0.029 \\
Duration & -0.643 \\
Attenuation coefficient & 0.081 \\
Constant & -1.592 \\
\hline
\end{tabular}

the coal mining MS signal. When the discriminant score is close to 1.442 , the signal will be considered as the blasting signal. Due to the only discriminant function, the obtained scores change in the $1 \mathrm{D}$ line, and the values of the coal mining MS signal and blasting signal are symmetric about the origin. Then the demarcation point of the scores could be considered as the origin and the discriminant process could be simplified. When the signal's discriminant score is less than zero, the signal will be determined as the coal mining MS signal, whereas the signal is considered as the blasting signal. The discriminant result is more reliable with the score larger or smaller than zero.

(4) Discriminant Function Coefficients of Coal Mining MS and Blasting Signals. Table 4 shows the discriminant function coefficients of coal mining MS and blasting signals through modeling. With $f_{r}$ representing the signal's dominant frequency, $t_{d}$ representing the duration, and $c_{a}$ referring to the attenuation coefficient, the discriminant function formula of the coal mining MS and blasting discriminant model could be expressed as follows:

$$
F\left(f_{r}, t_{d}, c_{a}\right)=0.029 f_{r}-0.643 t_{d}+0.081 c_{a}-1.592,
$$

where $F\left(f_{r}, t_{d}, c_{a}\right)$ refers to the discriminant score of the signal. If $F\left(f_{r}, t_{d}, c_{a}\right)<0$, the signal is determined as the coal mining MS signal; if $F\left(f_{r}, t_{d}, c_{a}\right)>0$, the signal will belong to the blasting signal. The discriminant result is more reliable with $F\left(f_{r}, t_{d}, c_{a}\right)$ larger or smaller than zero.

4.3. Performance of the Discriminant Model. Table 5 shows the eigenvalues of the discriminant analysis. The eigenvalues represent the amount of information, so the larger the eigenvalue, the higher the discriminating performance of the function. The canonical correlation coefficient refers to 


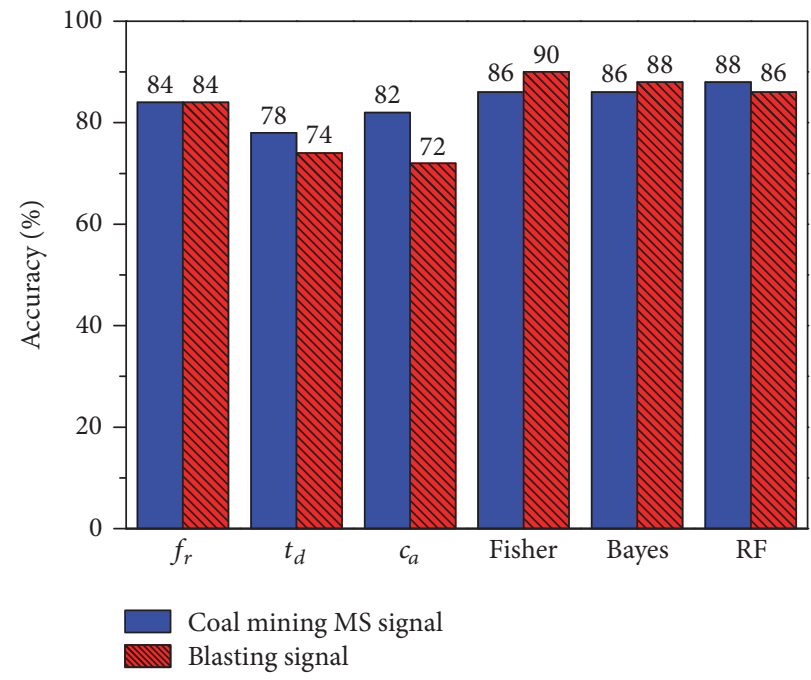

FIGURE 8: Comparison of discriminant results of three classifiers and single characteristic.

TABLE 5: Eigenvalue of discriminant analysis.

\begin{tabular}{lcccc}
\hline Function & Characteristic value & Variance contribute rate\% & Cumulative variance contribute rate \% & Regular relativity \\
\hline 1 & 2.122 & 100.0 & 100.0 & 0.824 \\
\hline
\end{tabular}

TABLE 6: Identification results of training sample.

\begin{tabular}{lcccc}
\hline Category of signal & Total & Number of correct identifications & Number of error identifications & Correct recognition rate\% \\
\hline Coal mining MS & 50 & 45 & 5 & 90 \\
Blasting & 50 & 46 & 4 & 92 \\
\hline
\end{tabular}

the correlation between the function coefficients and the classified group. It could be seen from the table that the discriminant function capability is significant. The variance contribution rate is $100 \%$, which could explain $100 \%$ of the sample information, and the canonical correlation coefficient is higher.

The constructed coal mining MS and blasting discriminant model is utilized to test the signal data of 100 training samples, and the testing results are shown in Table 6. Among 50 groups of coal mining MS signals, 45 groups are recognized correctly, and 5 groups are recognized as the blasting signals mistakenly. Among 50 groups of blasting signals, 46 groups are recognized correctly, and 4 groups are wrongly recognized as the coal mining MS signals. Therefore, the correct recognition rates of the discriminant model for the coal mining MS signals and blasting signals are $90 \%$ and $92 \%$, respectively, which has relatively higher recognition rate than the single criterion.

To further check the performance of the discriminant model, 100 testing sample signals were identified and classified by using this model and then compared with the real category. Besides, two other classifiers (Bayes and Random forest (RF)) were applied to establish discriminant models using 100 training sample signals and check the accuracy of the discriminant models using 100 testing sample signals. The discriminant results of three classifiers for 100 testing sample signals were compared with the discriminant results using single characteristic. As shown in Figure 8, the discriminant results using multiple characteristic indexes are much better than the single indexes. The performance of the Fisher, Bayes, and RF will have little difference as long as the proper characteristic indexes are used, which indicates that selecting proper characteristic indexes is one of the key steps to distinguish different types of MS signals before establishing discriminant model. Take the discriminant results of Fisher as an example, among 50 groups of coal mining MS signals, 7 groups of signals are wrongly recognized as the blasting signals. Among 50 groups of blasting signals, 5 groups are wrongly recognized as the coal mining MS signals. It could be obtained that the correct recognition rates of the model for the coal mining MS signals and blasting signals are $86 \%$ and $90 \%$, respectively. The average accuracy of Fisher for two types of signals is $88 \%$, which is higher than the other two classifiers (the average accuracy are both 87\%). Thus, this Fisher discriminant model has relatively high accuracy and stability and its application could be promoted.

4.4. Application of the Discriminant Model. According to the analysis results of field MS monitoring at Qianqiu coal mine, a typical destructive mining MS event with energy more than $10^{7} \mathrm{~J}$ and a roof-break blasting test occurred at number 21141 longwall panel, respectively. Figures 9 and 10 are the waveforms of these MS events recorded by the 


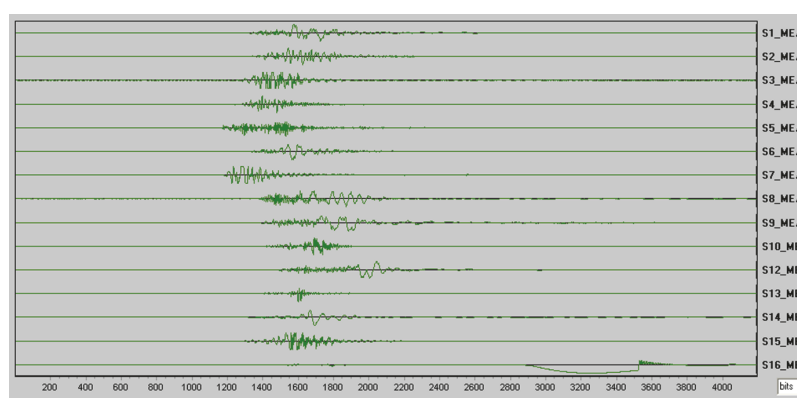

FIgURE 9: Waveforms of the destructive mining MS event.

ARAMIS MS monitoring system. As shown in Figure 9, there are 15 waveforms, among which, the waveform of S16 is not complete and its zero line drifts. Therefore, there are 14 waveforms involved in the source location. As shown in Figure 10, there are 16 waveforms, among which, S13 and S15 have low signal-noise ratio (SNR) and the arrival time and the terminal time could not be picked up. Therefore, the other 13 waveforms are involved in the source location.

The sensor with the earliest arrival time usually has the smallest travel distance from the source, and the recorded waveform has high SNR and is suitable for distinguishing the type of MS signals. For the destructive mining MS and blasting events, sensor S7 and sensor S3 had the earliest arrival time, and the signals of S7 and $\mathrm{S} 3$ were selected as the analysis object, respectively.

The analysis results of the destructive mining MS event are shown in Figure 11. The arrival time and the terminal time of sensor S7 are obtained by the STA/LTA method (the 1152th sampling point and the 2906th sampling point, resp.). Therefore the signal duration is $(2906-1152) / 500=$ $3.51 \mathrm{~s}$. Figure 11(b) is the spectrum of the signal, which was obtained by FFT method. It is found that the frequency distribution is dispersed. There are wideband signals with $10-250 \mathrm{~Hz}$ frequency. The dominant frequency is $37.76 \mathrm{~Hz}$ and the subdominant frequency and the third frequency are $86.7 \mathrm{~Hz}$ and $123.1 \mathrm{~Hz}$. As shown in Figure 11(c), the following peak envelope curve (red line) corresponds to the section from the 1344th sampling point to the 2906th sampling point. The declining process of the signal is not steady, which is the typical characteristic of the coal mining MS signals. The attenuation coefficient $(|b|)$ is 6.14 and the fitting precision (Adj. $R$-square) is 0.85 . The discriminant score is -2.26 , which means this is a coal mining MS event.

The analysis results of the blasting event are shown in Figure 12. The signal duration is $(1686-990) / 500=1.39 \mathrm{~s}$ (Figure 12(a)). Figure 12(b) is the spectrum of the signal, and the frequency distribution is relatively dispersed. There are obvious signals in $20-150 \mathrm{~Hz}$ frequency range. The dominant frequency is $135.97 \mathrm{~Hz}$. Figure 12(c) is the following peak envelope curve and attenuation curve of the signal. The following peak envelope curve (red line) corresponds to the section from the 1110th sampling point to the 1686th sampling point. The declining process of the signal is relatively steady and there are no obvious fluctuations. The attenuation coefficient $(|b|)$ is 9.04 and the fitting precision (Adj. $R$-square) is
0.96 . The discriminant score is 2.19 , indicating that the signal belongs to blasting event.

\section{Conclusions}

Three significant characteristics of the coal mining MS and blasting signals were studied by using FFT, STA/LTA, and envelope analysis. Based on these characteristics and Fisher method, a discriminant model for these two types of signals was established. The conclusions are as follows.

(1) The following peak attenuation curves of the mining MS and blasting signals satisfy the power function, which makes it reasonable to quantify the attenuation and stability of the signals utilizing the corresponding fitting coefficient and fitting precision values. Generally, the fitting precision and the attenuation coefficient of the coal mining MS are smaller than that of the blasting signals, which means that the attenuation of the coal mining MS signals is slower and more fluctuant than that of the blasting signals.

(2) Waveform characteristics consisting of the dominant frequency, the duration, and the attenuation coefficient were extracted as the discriminating parameters. And the boundaries between the two types of signals on the three characteristics were obtained. At the same time, the discriminating performance of the three characteristics was compared and discussed. The dominant frequency is most significant in distinguishing between two types of signals followed by duration. The attenuation coefficient's impact is relatively minimal.

(3) Based on the waveform characteristics, a discriminant model of coal mining MS and blasting signals was established using Fisher method. Its performance was checked and compared with other methods by using the real data from Qianqiu coal mine. Results show that the correct discriminant rate of the coal mining MS and blasting signals in the testing samples is 86 percent and 90 percent, which can basically meet the requirements of the MS monitoring data processing at the coal mine site. Besides, results also demonstrated that the identification results using multiple characteristic indexes are much better than the single indexes. The performance of different recognition methods such as the Fisher method, Bayes method, and Random Forest will have little difference as long as the proper characteristic indexes are used, which indicates that selecting proper characteristic indexes is one of the key steps to distinguish different types of MS signals before establishing discriminant model.

(4) The results are useful to improve the efficiency of automatic recognition for coal mining MS signals and blasting signals and reduce the workload of artificial recognition. Although the established discriminant model in this paper is based on the monitoring data in Qianqiu coal mine, the research results could be 


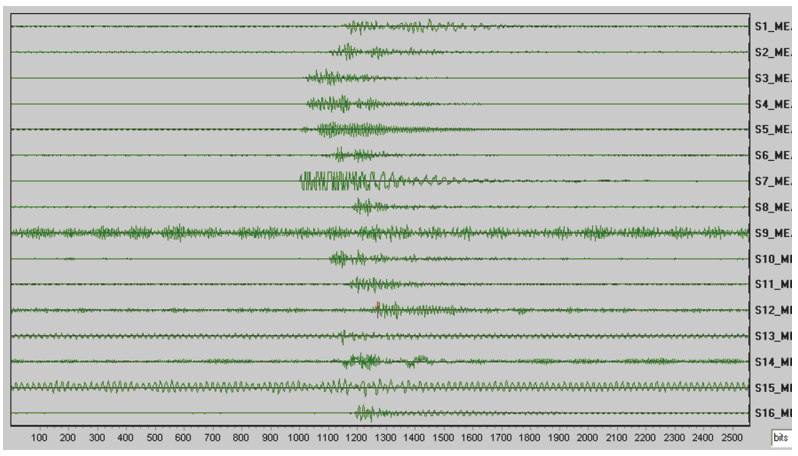

FIgURE 10: Waveforms of the blasting event.

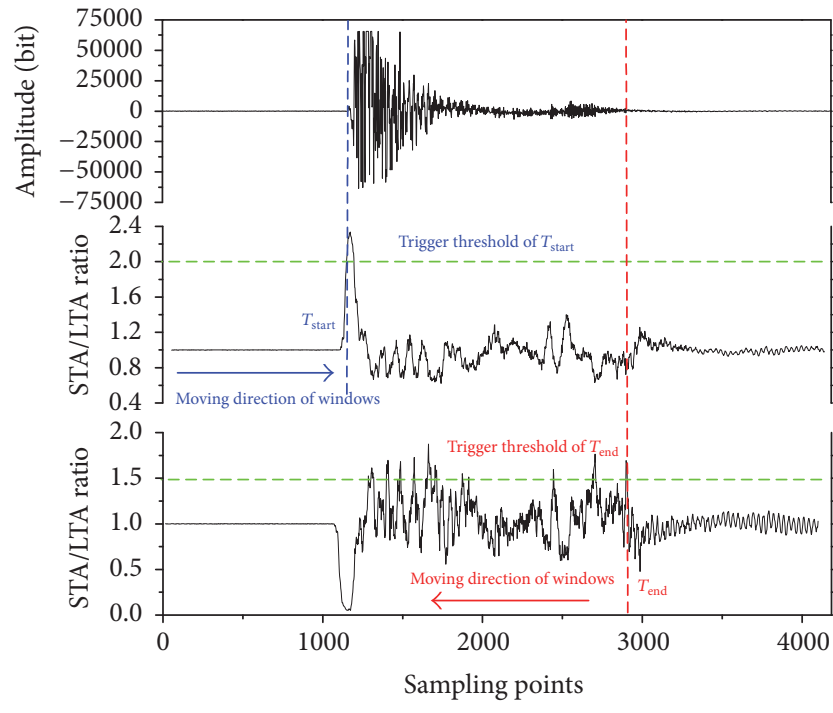

(a) Duration of signal

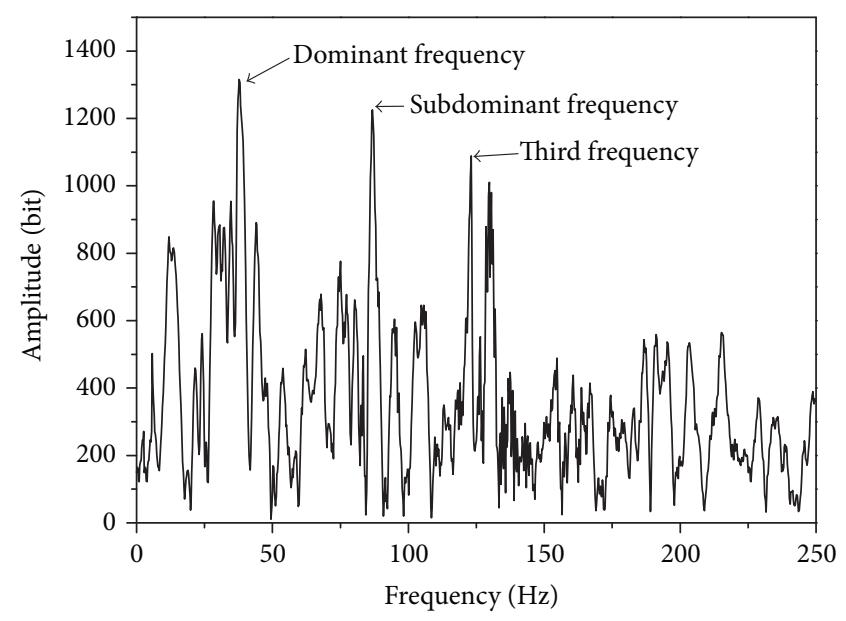

(b) Spectrum of signal

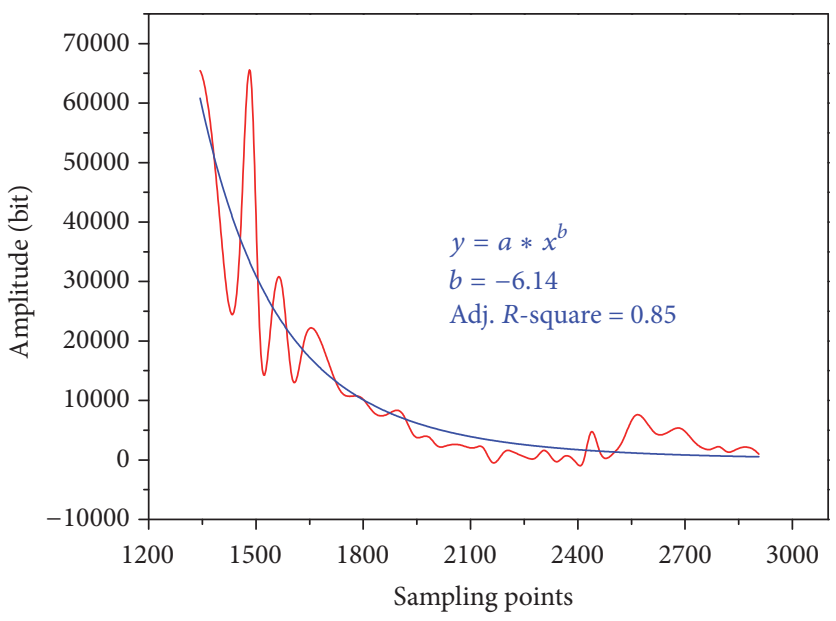

- Envelope curve

- Attenuation curve

(c) Attenuation of signal

FIGURE 11: Signal characteristics of sensor S7 for the destructive mining MS event. 


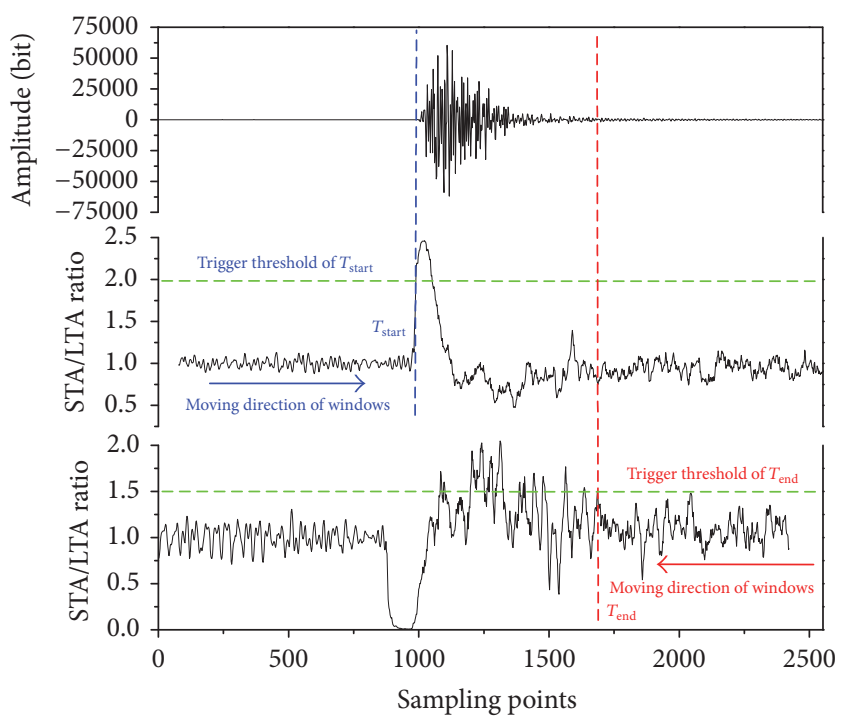

(a) Duration of signal

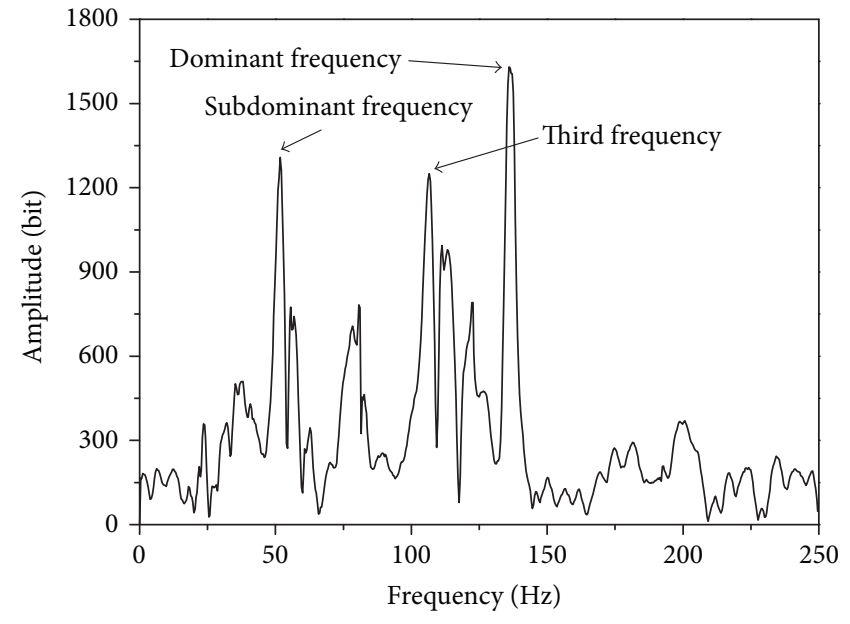

(b) Spectrum of signal

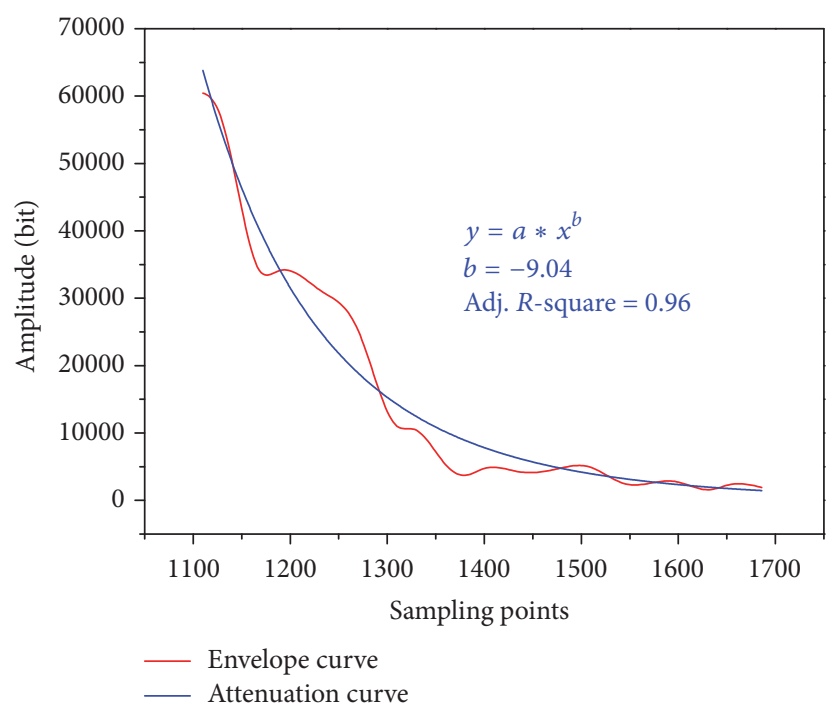

(c) Attenuation of signal

FIGURE 12: Signal characteristics of sensor S3 for the blasting event.

utilized for other mines and tunnel engineering to a great extent by adjusting the parameters.

\section{Conflicts of Interest}

The authors declare that they have no conflicts of interest.

\section{Acknowledgments}

This work is supported by the National Natural Science Foundation of China (51504250), the Natural Science Foundation of Jiangsu Province (BK20150183), the National Natural Science Foundation of China (Key Program, 51634001), State Key Laboratory of Coal Resources and Mine Safety of China (no. SKLCRSM14X03), and the Fundamental Research Funds for the Central Universities (2015QNA58).

\section{References}

[1] L. M. Dou, Z. L. Mou, and C. P. Lu, Theory and technology of mining geophysics, Science Press Ltd, Beijing, China, 2014.

[2] Q. X. Qi, Z. H. Ouyang, S. K. Zhao et al., "Study on types of rock burst mine and prevention methods," Coal Science and Technology, vol. 42, no. 10, pp. 1-5, 2014.

[3] Y. Jiang and Y. Zhao, "State of the art: Investigation on mechanism, forecast and control of coal bumps in China," Chinese Journal of Rock Mechanics and Engineering, vol. 34, no. 11, pp. 2188-2204, 2015.

[4] T. H. Ma, C. A. Tang, L. X. Tang, W. D. Zhang, and L. Wang, "Rockburst characteristics and microseismic monitoring of deep-buried tunnels for Jinping II Hydropower Station," Tunnelling and Underground Space Technology, vol. 49, pp. 345368, 2015. 
[5] M. Ge, "Efficient mine microseismic monitoring," International Journal of Coal Geology, vol. 64, no. 1-2, pp. 44-56, 2005.

[6] L. Gulia, "Detection of quarry and mine blast contamination in European regional catalogues," Natural Hazards, vol. 53, no. 2, pp. 229-249, 2010.

[7] D. Malovichko, "Discrimination of blasts in mine seismology," in Proceedings of the in Proceeding of the Deep Mining, Australian Centre for Geomechanics, Perth, Australia, 2012.

[8] L. Dong, J. Wesseloo, Y. Potvin, and X. Li, "Discrimination of mine seismic events and blasts using the fisher classifier, naive bayesian classifier and logistic regression," Rock Mechanics and Rock Engineering, vol. 49, no. 1, pp. 183-211, 2016.

[9] C. Zeiler and A. A. Velasco, "Developing local to near-regional explosion and earthquake discriminants," Bulletin of the Seismological Society of America, vol. 99, no. 1, pp. 24-35, 2009.

[10] J. L. Bonner, A. Stroujkova, and D. Anderson, "Determination of Love-and Rayleigh-wave magnitudes for earthquakes and explosions," Bulletin of the Seismological Society of America, vol. 101, no. 6, pp. 3096-3104, 2011.

[11] W. Kim, D. W. Simpson, and P. G. Richards, "Discrimination of earthquakes and explosions in the eastern United States using regional high-frequency data," Geophysical Research Letters, vol. 20, no. 14, pp. 1507-1510, 1993.

[12] D. Fäh and K. Koch, "Discrimination between earthquakes and chemical explosions by multivariate statistical analysis: A case study for Switzerland," Bulletin of the Seismological Society of America, vol. 92, no. 5, pp. 1795-1805, 2002.

[13] M. AllamehZadeh, "Discrimination analysis of earthquakes and man-made events using ARMA coefficients determination by artificial neural networks," Natural Resources Research, vol. 20, no. 4, pp. 367-375, 2011.

[14] Y. J. Bian, H. M. Huang, and T. T. Wang, "A research on the SVM classification of earthquake and explosion based upon seismic wave features," International Journal of Digital Content Technology and Its Applications, vol. 26, no. 5, pp. 888-898, 2014.

[15] L.-J. Dong, J. Wesseloo, Y. Potvin, and X.-B. Li, “Discriminant models of blasts and seismic events in mine seismology," International Journal of Rock Mechanics \& Mining Sciences, vol. 86, pp. 282-291, 2016.

[16] J. Ma, G. Y. Zhao, L. J. Dong et al., "A Comparison of mine seismic discriminators based on features of source parameters to waveform characteristics," Shock and Vibration, vol. 2015, Article ID 919143, 10 pages, 2015.

[17] G.-Y. Zhao, J. Ma, L.-J. Dong, X.-B. Li, G.-H. Chen, and C.-X. Zhang, "Classification of mine blasts and microseismic events using starting-up features in seismograms," Transactions of Nonferrous Metals Society of China, vol. 25, no. 10, pp. 34103420, 2015.

[18] J. A. Vallejos and S. D. McKinnon, "Logistic regression and neural network classification of seismic records," International Journal of Rock Mechanics and Mining Sciences, vol. 62, pp. 8695, 2013.

[19] G.-Y. Zhao, Q.-L. Deng, and J. Ma, "Recognition of mine microseismic signals based on FSWT time-frequency analysis," Chinese Journal of Geotechnical Engineering, vol. 37, no. 2, pp. 306-312, 2015.

[20] Y. S. Pan, X. F. Lv, and Z. H. Li, "The model of energy absorbing coupling support and its application in rockburst roadway," Journal of Mining and Safety Engineering, vol. 28, no. 1, pp. 6165-65, 2011.
[21] Q. J. Zhu, F. X. Jiang, Y. M. Yin et al., "Classification of mine microseismic events based on wavelet-fractal method and pattern recognition," Chinese Journal of Geotechnical Engineering, vol. 34, no. 11, pp. 2036-2042, 2012.

[22] F.-X. Jiang, Y.-M. Yin, Q.-J. Zhu, S.-X. Li, and Z.-X. Yu, "Feature extraction and classification of mining microseismic waveforms via multi-channels analysis," Journal of Coal Science \& Engineering (China), vol. 39, no. 2, pp. 229-237, 2014.

[23] X. Li, Z. Li, E. Wang et al., "Analysis of natural mineral earthquake and blast based on Hilbert-Huang transform (HHT)," Journal of Applied Geophysics, vol. 128, pp. 79-86, 2016.

[24] C.-P. Lu, L.-M. Dou, B. Liu, Y.-S. Xie, and H.-S. Liu, "Microseismic low-frequency precursor effect of bursting failure of coal and rock," Journal of Applied Geophysics, vol. 79, pp. 55-63, 2012.

[25] B. Kong, E. Wang, Z. Li et al., "Electromagnetic radiation characteristics and mechanical properties of deformed and fractured sandstone after high temperature treatment," Engineering Geology, vol. 209, pp. 82-92, 2016.

[26] K. W. Cattermole, "The Fourier transform and its applications," Electronics and Power, vol. 11, no. 10, p. 357, 2009.

[27] C. Baillard, W. C. Crawford, V. Ballu, C. Hibert, and A. Mangeney, "An automatic kurtosis-based P-and S-phase picker designed for local seismic networks," Bulletin of the Seismological Society of America, vol. 104, no. 1, pp. 394-409, 2014.

[28] I. V. Rodriguez, "Automatic time-picking of microseismic data combining STA/ LTA and the stationary discrete wavelet transform," CSPG CSEG CWLS Convention, pp. 1-4, 2011.

[29] H. Liu and J. Z. Zhang, "STA/LTA algorithm analysis and improvement of Microseismic signal automatic detection," Progress in Geophysics, vol. 29, no. 4, pp. 1708-1714, 2014.

[30] X. F. Jia, H. Q. An, and S. G. Zhang, "Cubic spline interpolation method for the envelope tracking of middle and low frequency voltage flicker," Advanced Materials Research, vol. 960-961, pp. 704-709, 2014.

[31] K. S. Sim, M. A. Kiani, M. E. Nia, and C. P. Tso, "Signal-to-noise ratio estimation on SEM images using cubic spline interpolation with Savitzky-Golay smoothing," Journal of Microscopy, vol. 253, no. 1, pp. 1-11, 2014.

[32] M.-S. Gao, L.-M. Dou, N. Zhang, Z.-L. Mu, K. Wang, and B.-S. Yang, "Experimental study on earthquake tremor for transmitting law of rockburst in geomaterials," Chinese Journal of Rock Mechanics and Engineering, vol. 26, no. 7, pp. 1365-1371, 2007.

[33] L.-J. Dong, X.-B. Li, and Y.-F. Bai, "Fisher discriminant analysis model for classifying top coal cavability of the steep seam," Journal of the China Coal Society, vol. 34, no. 1, pp. 58-63, 2009.

[34] L. J. Dong, X. B. Li, G. Y. Zhao et al., "Fisher discriminant analysis model and its application to predicting destructive effect of masonry structure under blasting vibration of openpit mine," Chinese Journal of Rock Mechanics and Engineering, vol. 28, no. 4, pp. 750-758, 2009. 


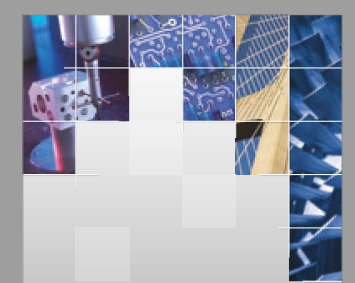

\section{Enfincering}
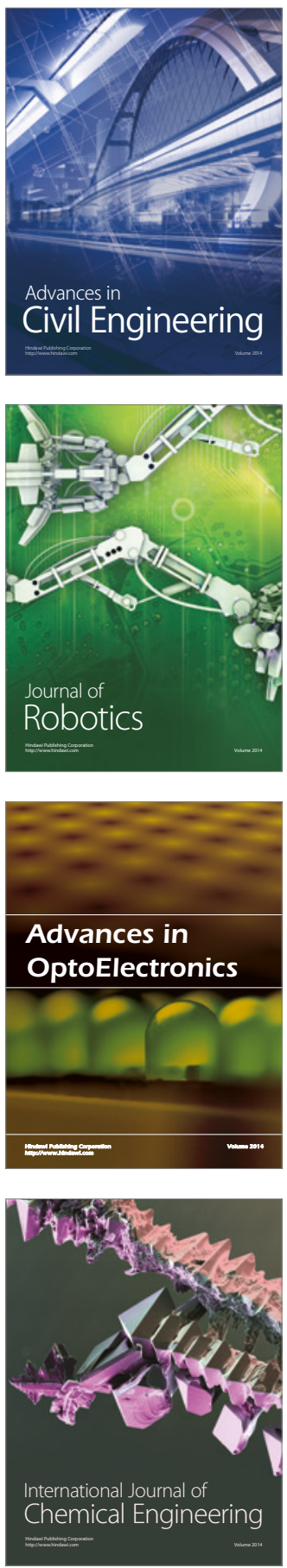

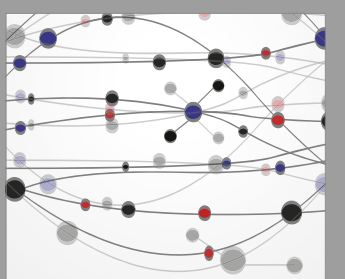

The Scientific World Journal

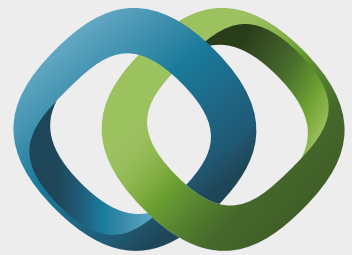

\section{Hindawi}

Submit your manuscripts at

https://www.hindawi.com
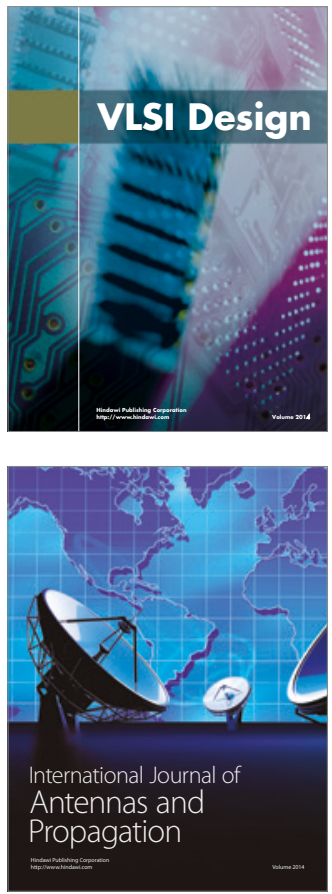

\section{Rotating}

Machinery
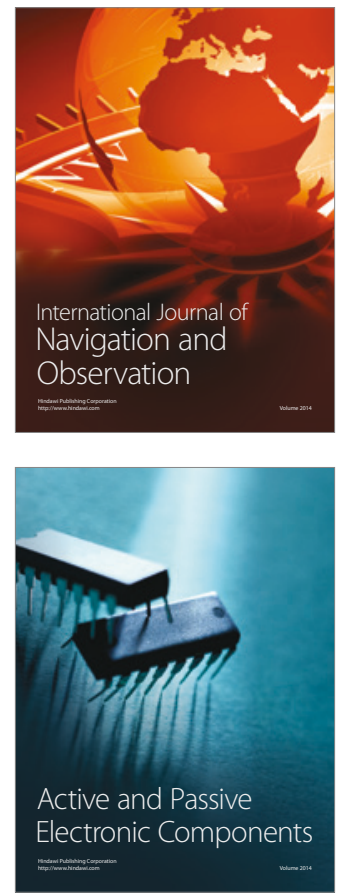
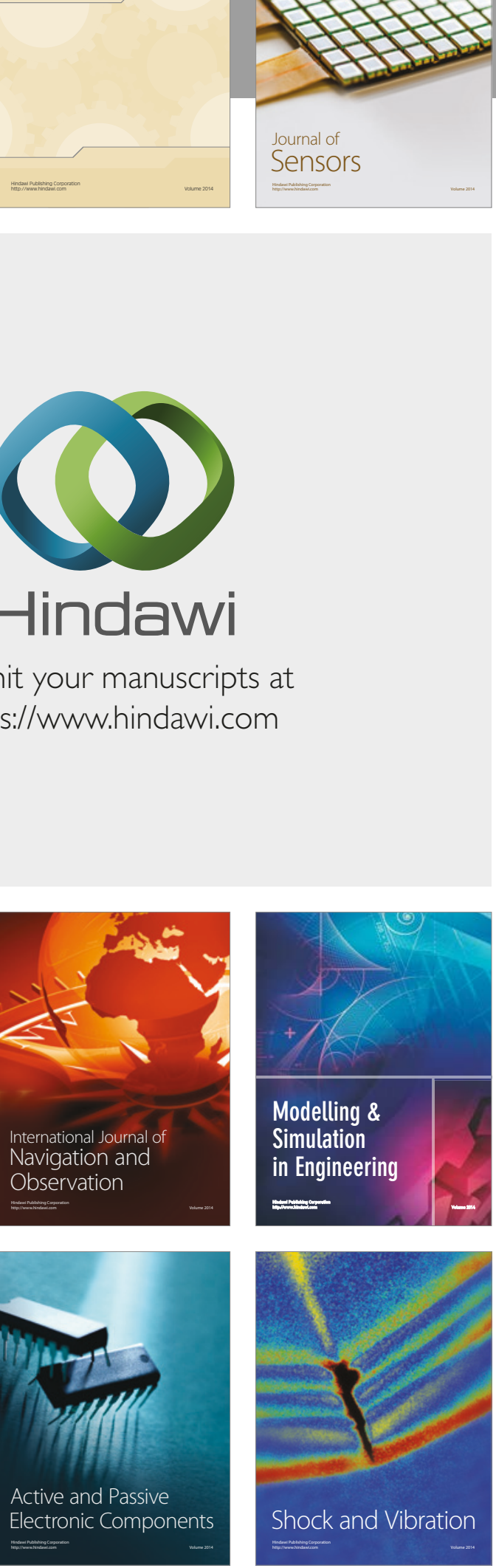
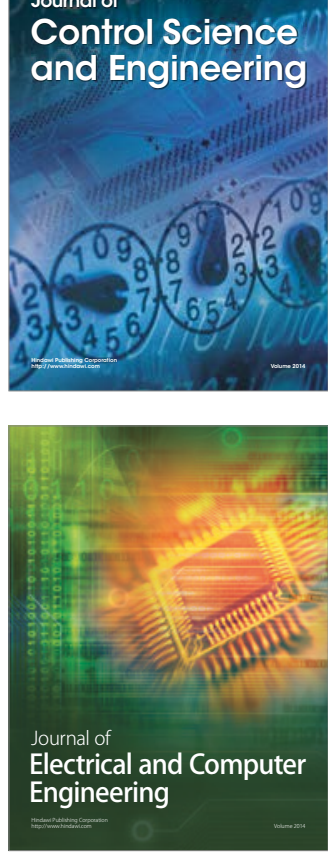

Distributed

Journal of

Control Science

and Engineering
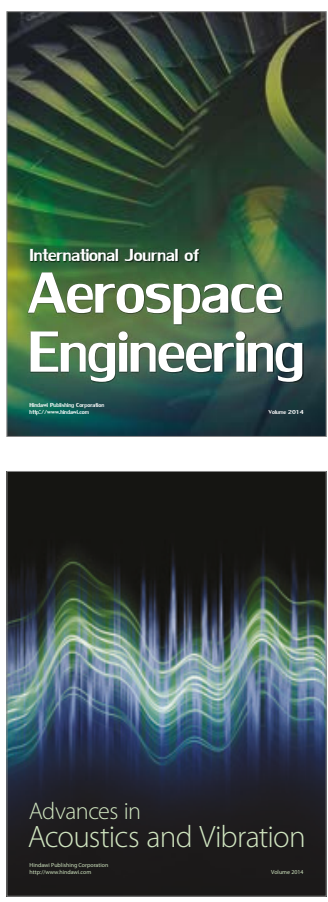

Sensor Networks 